\title{
Initial Community Hospital Performance of Radioactive Seed Localization for Breast Cancer Patients
}

\author{
James A Wheeler ${ }^{1} \&$ Fang Liu $^{2}$ \\ ${ }^{1}$ Goshen Center for Cancer Care, United States \\ ${ }^{2}$ University of Notre Dame, United States \\ Correspondence: James A Wheeler, Goshen Center for Cancer Care, United States. \\ E-mail: jwheeler@goshenhealth.com \\ Received: December 18, $2016 \quad$ Accepted: January 25, $2017 \quad$ Online Published: February 12, 2017 \\ doi:10.5539/cco.v6n1p51 \\ URL: http://dx.doi.org/10.5539/cco.v6n1p51
}

\begin{abstract}
Purpose: To compare the positive margin rates for women with nonpalpable breast tumors who underwent breast conserving surgery with wire localization versus those with radioactive seed localization in a small community hospital and to compare the size of the corresponding breast specimens.
\end{abstract}

Introduction: Wire localization (WL) has been the standard technique to assist in the removal of nonpalpable breast tumors for the past three decades for patients undergoing breast conserving surgery. Radioactive seed localization (RSL) is an alternative technique that provides advantages of patient comfort and scheduling convenience. There are numerous studies from large academic centers, but little information on how successfully this technique can be implemented in community hospitals.

Methods: Thirty-five patients who underwent WL between September 18, 2013 and December 10, 2014 were compared to 110 patients who underwent RSL between February 12, 2014 and December 16, 2015.

Results: Three of the $35 \mathrm{WL}$ patients $(8.5 \%)$ had a positive margin compared to 14 of the 110 RSL patients $(12.7 \%)$, but this difference was not statistically significant (p-value 0.763 ). The breast specimen weight had a geometric mean of $30.26 \mathrm{~g}$ for the WL patients and $32.78 \mathrm{~g}$ for the RSL patients, a difference of $8.3 \%$, which was not statistically significant. Positive margin rates did not depend on the surgeon or the radiologist placing the I-125 localization seed.

Conclusion: The RSL technique can be implemented in community hospitals with the expectation of having the same positive margin rates as reported from academic centers.

Keywords: Breast conserving surgery (BCS), Radioactive seed localization (RSL), Wire localized breast biopsies (WL)

\section{Introduction}

Breast conserving surgery in conjunction, when appropriate, with adjuvant radiation therapy, has been established to provide equivalent survival as a mastectomy for women with early stage breast cancer (Darby, McGale, \& Correa, 2011).

Wire localization has been the standard radiologic technique to assist in the removal of nonpalpable breast tumors for the past three decades (Homer \& Pile-Spellman, 1986). Wire localization (WL) uses a needle-wire assembly under image guidance to place the tip of the marker wire in the tumor for subsequent surgical removal. Depending on the clinical situation, it can now be performed under mammographic, ultrasound, or magnetic resonance image guidance.

The WL technique requires close coordination between the radiologist, surgeon, and operating room schedules. The need to avoid patient delay for her scheduled arrival in the operating room places pressure on the radiology team. The problem of potential wire migration between the time it is inserted in the radiology suite and subsequent surgical removal in the operating room has been known for over 25 years (Davis, Wechsler, Feig, \& March, 1988). The guide-wire can break. The surgeon may remove the lesion in continuity with the localization wire rather than choose a better position of skin entry, with resultant unnecessary loss of normal breast tissue remote from the tumor. 
The radioactive seed localization (RSL) technique, which will be described in greater detail in the discussion section, was first described in 2001 from the H. Lee Moffit Cancer Center (Gray et al., 2001). A low energy I-125 (27-keV) source is inserted into the breast lesion prior to the surgery by the radiologist under image guidance. The surgeon then removes the breast mass at the appropriate time using a radioactive probe (Jakub et al., 2010). The specimen radiograph and radiation survey measurements of the patient in the operating room confirm that all the radioactive material has been removed. The RSL technique requires careful handling of the radioactive materials and corresponding documentation (Pavlicek, Walton, Karstaedt, \& Gray, 2006). The operating room staff and the pathology staff likewise need to follow special procedures as directed by the radiation safety officer (Graham, Jakub, Brunette, \& Reynolds, 2012).

We initiated our RSL program February 12, 2014 with one breast surgeon and two diagnostic radiologists trained in this technique. We reported on our initial 2014 experience comparing 48 RSL patients and 36 WL patients from our community hospital (Wheeler et al., 2016). Our initial positive margin rate was not statistically different between the WL $(8.3 \%)$ and RSL (10.4\%) patients, but the specimen weight was larger for the RSL patients $(63 \mathrm{~g})$ than for the WL patients $(33 \mathrm{~g} ; \mathrm{p}=0.02)$. If the size difference persisted with a significantly larger number of patients, there could be legitimate concerns that the cosmetic outcome was compromised using the RSL technique. Since many factors influence specimen size and there is inevitably a learning curve with any surgical technique, we chose to carefully evaluate our RSL program during its second year.

Our previous work did not contain information regarding hormonal receptor status. A recent Chinese report indicated that patients with Her-2 positive breast cancer had an increased risk of positive margins following breast conserving surgery (Jia $\mathrm{H}$ et al., 2014). We wished to see if Her-2 status was similarly associated with positive margins among our patients.

Since many of our patients had their screening mammograms and core biopsy at an outside institution, we wondered if patients diagnosed at our hospital had a shorter delay between initial imaging finding and breast conserving surgery or between core biopsy and breast conserving surgery. For these reasons, we embarked upon this new study.

\section{Method}

\subsection{Data Collection}

The study institution is an approximately 100 bed community hospital located in a rural community of 32,000. The hospital IRB gave approval to conduct this retrospective study. Through the tumor registry, 60 patients were identified who underwent breast conserving surgery (BCS) between September 18, 2013 and December 10, 2014. Thirty-eight patients had nonpalpable tumors and underwent BCS using WL. Two patients had benign disease and were excluded from further analysis. One patient with invasive cancer received pre-operative chemotherapy and was likewise excluded. The remaining 35 patients constituted the WL group. The WL patients treated by surgeons who chose to not learn the RSL technique were included through December 2014. As the RSL program matured, the two surgeons who performed the most BCS cases used WL only if there were features that made them unsuitable for RSL. Consequently, the WL patients after 2014 were not included in this study.

The RSL patients were identified through the nuclear medicine records. Patients who had benign disease or who had preoperative chemotherapy were excluded. The 110 RSL patients included in this study underwent BCS between February 12, 2014 and December 16, 2015.

\subsection{Study Objectives}

The primary objective was to determine if the introduction of the RSL technique significantly changed the positive margin rate compared to the WL technique. Secondary objectives included determining if the weight of the breast specimen removed was significantly different between the RSL and WL techniques. We also wished to determine if the positive margin rate differed among the surgeon or among the radiologist who placed either the localization wire or the radioactive seed. We sought to determine if the positive margin rate depended on the estrogen receptor, progesterone receptor, or Her-2 receptor status. We evaluated the positive margin rate by the DCIS size, DCIS grade, invasive tumor size, invasive tumor grade, or patient age.

\subsection{Statistical Analysis}

The Fisher exact test was used to compare the margin positivity rate between the WL and RSL techniques. The p-value and the odds ratio of having a positive margin between the RSL and WL techniques were calculated. The logistic regression model was applied to explore the relationship between the likelihood of having a positive margin and the patient age, weight of the breast specimen, receptor status, the DCIS size, DCIS grade, invasive tumor size, invasive tumor grade, surgeon, radiologist, and other factors. The 2-sample t-test was performed to 
compare the log-transformed time from initial imaging finding to biopsy for imaging performed at an outside institution compared to inside the institution, and to compare the log-transformed time from core biopsy to breast conserving surgery for patients diagnosed outside or within the institution.

\section{Results}

\subsection{Patients}

Thirty-five patients underwent wire localization BCS between September 18, 2013 and December 10, 2014. The comparison group consisted of 110 RSL patients who underwent BCS between February 12, 2014 and December 16, 2015. Table 1 demonstrates the patient characteristics of both groups in terms of tumor stage and the patient age at the time of BCS. As expected for patients with nonpalpable breast lesions, over half the patients had stage 0 or I tumors. The patients in the WL group had an average age of 65 years compared to 62 years for the RSL patients.

\subsection{Source Placement Relative to Breast Conserving Surgery}

Of the 110 patients who underwent RSL, only one patient had two I-125 seeds. The remainder all had a single I-125 seed placed. The majority of patients had the I- 125 seed placed two days prior to surgery, with a range of 0 to 28 days. Eight patients had seed placement the same day as BCS. Two patients had the seed placement one day prior to BCS. Seventy-one patients had seed placement two days prior to BCS. Nine patients had seed placement three days prior to BCS, two patients four days, and four patients five days prior to BCS. Although our program was designed to have BCS within five days of I-125 seed placement, individual circumstances resulted in four patient having BCS more than seven days after seed placement. The greatest delay came from a patient who cancelled her planned surgery to seek a second surgical opinion at a different facility, but ultimately returned for BCS 28 days following I-125 placement.

\subsection{Positive Margin Rates}

Three of the $35 \mathrm{WL}$ patients (8.5\%) had a positive margin compared to 14 of the $110 \mathrm{RSL}$ patients (12.7\%). The odds of having a positive margin in patients who underwent RSL were 1.55 times the odds in patients who had WL, with a $95 \%$ CI of $(0.396,8.950)$. The p-value (Fisher's exact test) was 0.763 , indicating the difference in the margin positivity rate was not statistically significant.

Surgeon A performed 127 of the cases, Surgeon B performed 17 cases, and Surgeon C performed 1 case among the 145 total WL and RSL patients. We combined the cases of the last two surgeons to form a group of 18 patients. The odds of having a positive margin for Surgeon A was 0.622 times of the odds of the combined patients of the other two surgeons, with a $95 \%$ CI of $(0.147,3.760)$. The p-value (Fisher's exact test) was 0.445 . The margin positivity rate did not depend on whether Surgeon A or the combined group of Surgeons B and C performed the BCS in this combined group of WL and RSL patients in a statistically significant manner.

The three radiologists who performed RSL also performed all but five of the WL procedures. Combining the four radiologists who performed these five cases into a single group formed the reference category, which had no positive margin cases. There was no statistically significant difference in the margin positivity rate based on the radiologist placing the wire or radioactive localization seed, as shown in table 2.

Separating the analysis to include only the 110 RSL patients, Surgeon A performed 97 cases and Surgeon B performed 13 cases. The odds ratio of having a positive margin for Surgeon A versus Surgeon B was 1.212 $(0.368,4.684$ with a p-value $>0.999$.

For the three radiologists who performed the 110 RSL cases, Radiologist A performed 21 cases with 3 positive margins, Radiologist B performed 39 cases with 3 positive margins, and Radiologist $\mathrm{C}$ performed 50 cases with 8 positive margins. There was no association between the radiologist and the positivity of the margins ( $\mathrm{p}$-value $=$ 0.492).

Although there was no association between the surgeon or radiologist involved in the RSL procedure and the positive margin rate, this is partly due to the overall low positive margin rate and the number of patients in this study. A power calculation shows that, for two equally sized groups of surgeons (or radiologists), if the positive margin rate for group 1 is $10 \%$ and for group 2 is $15 \%$, to reach $80 \%$ power with $5 \%$ Type- 1 error rate requires 685 patients per group. If the positive margin rate for group 1 is $10 \%$ and for group 2 is $20 \%$, to reach $80 \%$ power with 5\% Type-1 error rate requires 200 subjects per group. The same concern applies to the type of surgical procedure performed, namely WL versus RSL.

\subsection{Time Interval from Abnormal Mammogram to Biopsy}

To compare the time interval between the abnormal mammogram and the biopsy for patients who had their 
mammogram at our institution compared to other locations, the two-sample t-test was performed on the log-transformed time interval. The geometric mean (days) was 15.1 days at our location versus 14.6 days for other locations. This represented a 3.3\% change. The 95\% CI was $(-28.9,50.0) \%$; with a p-value of 0.864 .

\subsection{Time Interval from Abnormal Mammogram to Breast Conserving Surgery}

The two-sample t-test was used to compare the log-transformed time interval between biopsy to breast conserving surgery for patients whose abnormal imaging was performed at the study institution compared to other locations. The geometric mean was 23.0 days for patients imaged at the study institution versus 35.1 days for those imaged elsewhere. This represented a $-34.5 \%$ change. The $95 \%$ CI was $(-47.6,-18.1) \%$, p $<0.001$.

\subsection{Covariates}

\subsubsection{Specimen Weight of RSL versus WL}

The two-sample t-test was used to compare the log-transformed specimen weight between patients undergoing WL versus RSL. The geometric mean was $32.78 \mathrm{~g}$ for the RSL patients versus $30.26 \mathrm{~g}$ for the WL patients, a difference of $8.3 \%$. The $95 \%$ CI was $(-17.2,41.6) \%$ with a p-value of 0.553 . There was no statistically significant difference in the specimen weight of the RSL and WL patients.

\subsubsection{Invasive Tumor Grade and Tumor Size}

To determine whether tumor grade or size influenced the positive margin rate for either the invasive or DCIS components, a logistic regression was performed with the Firth bias correction. Compared to grade 1 patients with invasive cancer, the odds ratio for a positive margin for grade 2 patients was 0.602 with a $95 \%$ CI $(0.159$, 2705); p-value 0.481. Compared to grade 1 patients, for grade 3 patients with invasive cancer the odds ratio for a positive margin was 0.458 with a $95 \% \mathrm{CI}(0.086,2.423)$; $\mathrm{p}$-value $=0.346$.

When the invasive tumor size increased by $1 \mathrm{~mm}$, the odds of having a positive margin increased by $7.2 \%$ with a $95 \% \mathrm{CI}$ of $(2.0,13.6) \%$; the p-value $=0.006$.

\subsubsection{DCIS Size and Grade}

Whether the DCIS size influenced the positive margin rate was likewise examined via logistic regression with Firth bias correction. The odds ratio of having a positive margin was 1.053 with a $95 \%$ CI of $(1.012,1.097)$ if the DCIS size increased by $1 \mathrm{~mm}$, and the associated p-value was 0.012 .

Regarding the possible effect of DCIS grade on the positive margin rate with grade 1 as the reference category, the odds ratio of having a positive margin for grade 2 DCIS patients was 0.603 with a $95 \%$ CI of $(0.160,0.463)$; p-value 0.463 . For grade 3 DCIS patients compared to grade 1 patients, the odds ratio for having a positive margin was 0.301 with a $95 \%$ CI of $(0.048,1.514)$; p-value 0.145 .

The odds of having a positive margin in patients with a DCIS component was 1.21 times of the odds in patients without, with a $95 \%$ CI of $(0.368,4.684)$. The p-value of the Fisher's exact test was $>0.999$. This difference was not statistically significant.

\subsubsection{Size of Invasive Tumor or DCIS and Specimen Weight}

There was a positive correlation between the invasive tumor size and the specimen weight for the patients with invasive breast cancer (Spearman correlation coefficient $=0.331$; p-value $<0.001$ ). There was not a similar positive correlation between the DCIS size and the specimen weight for the patients with DCIS and without an invasive component (stage 0). The Spearman correlation coefficient was 0.06; p-value 0.640.

\subsubsection{Patient Age}

Whether age influenced the positive margin rate was examined via logistic regression with the Firth bias correction. The odds ratio of having a positive margin was 1.041 with a $95 \%$ CI of $(0.994,1.094)$ if the age increased by 1 year; the associated p-value was 0.088 .

\subsubsection{Estrogen Receptor Status}

All 17 of the positive margins occurred in the 136 patients with estrogen receptor (ER) positive invasive tumors or DCIS. None of the 9 ER negative patients had a positive margin, but this difference was not statistically significant. The odds ratio of having a positive margin between ER negative and positive patients was 0 . The associated $95 \% \mathrm{CI}$ was $(0,3.92)$, with a p-value 0.599 .

\subsubsection{Progesterone Receptor Status}

Among the 126 patients with progesterone receptor (PR) positive invasive cancer or DCIS, 13 had positive margins. Of the 19 PR negative patients, four had a positive margin. The odds of having a positive margin in PR 
negative patients was 2.30 times of the odds in PR positive patients. The associated 95\% CI was $(0.48,8.83)$ with a p-value 0.241 .

\subsubsection{Her-2 Copy Number}

Whether the Her-2 copy number influenced the positive margin rate was examined via the logistic regression with the Firth bias correction. The odds ratio of having a positive margin was 0.998 if the Her- 2 copy number increased by 0.1 unit with a $95 \% \mathrm{CI}$ of $(0.935,1.021)$. The p-value was 0.899 .

\section{Discussion}

The use of wire localization to assist the surgeon remove nonpalpable breast tumors has been used for the past three decades. Its disadvantages include the need for close coordination between the radiologist inserting the localization wire and the operating room and surgeon for breast tumor removal the same day. The localization wire can be dislocated from its correct position, and the patient has a wire protruding from her breast until the operative procedure.

With the RSL approach, the radiologist places a low activity I-125 source, typically with an activity between 0.100 and $0.289 \mathrm{mCi}(3.7-10.7 \mathrm{MBq})$ into the breast under image guidance, generally using either ultrasound or mammography. Since the technique of placing the I-125 source is similar to the placement of a radiographic marker immediately following a breast biopsy, it is relatively straightforward for the radiologist to learn the I-125 technique.

Just as a set of mammographic images are obtained immediately following a breast biopsy to document the biopsy location in the breast, mammographic images are obtained to document the I-125 seed placement. If the I-125 seed is not in the correct location, then a second I-125 seed must be deployed and both I-125 seeds must be removed at the time of BCS. Clinically important seed migration is rare, constituting less than $1 \%$ of cases (McGhan, McKeever, \& Pockaj, 2011), (Barentsz, et al., (2013). Goudreau (Goudreau, Joseph, \& Seiler, 2015) provides an excellent description of the technique for the radiologist regarding inserting the I-125 seed.

The radiologist does need to obtain written consent from the patient for the placement of the radioactive seed, complete the written directive, perform radiation survey measurements, and assure that the patient obtains the appropriate instructions. Since the activity of the I-125 source is so low, the patient is free to conduct all of her normal activities (Dauer et al., 2013). She does not need to restrict her contact with other people.

The implanted I-125 source has a physical half-life of 60 days, so there is flexibility in the timing of the RSL source insertion relative to the planned breast conserving surgery. It can, as in wire localization, be placed the day of the surgery, as was the case for $8 / 110(7 \%)$ of our patients. The majority of our patients $(71 / 110=64 \%)$ had I-125 placement two days prior to the planned breast conserving surgery. Our program, like many others, intends for the surgical removal of the breast specimen to occur within five days of the I-125 seed placement. Sometimes exceptional circumstances arise leading to a delay in I-125 removal. Four of our patients had their BCS more than seven days from I-125 seed placement.

Since surgeons are familiar with the use of a radiation probe for performing a sentinel lymph node biopsy, learning the appropriate technique for localization of the I-125 seed placed within the tumor in the breast is relatively straightforward. The Technitium-99 used to assist in localizing the sentinel lymph node has a much higher energy $(140-\mathrm{keV})$ than that of the I-125 seed $(27-\mathrm{keV})$, but dual probes are available that allow the user to easily switch between the energies of these two isotopes (Aima, Viscariello, Patton, \& Bednarz, 2016).

Since breast tissue is normally pliable, the likelihood of damaging the I-125 with a scalpel during removal of the breast specimen is remote because the seed tends to be pushed to one side. A study in pig tissue demonstrated that the I-125 seed had to be immobilized with tweezers before it could be damaged with either a scalpel or an electrocautery blade (Classic, Brunette, \& Carlson, 2009).

The standard surgical radiograph used to evaluate the breast specimen also serves to confirm removal of the I-125 seed. It is still necessary to perform a radiation survey of the patient to further confirm and document removal of all radioactive material. If a seed was transected and a portion remained in the patient, this radiation survey should alert the surgeon to take appropriate action.

The pathology team also must learn the correct handling of the breast specimen containing the I-125 seed (Graham, Jakub, Brunette, \& Reynolds, 2012). It is possible to transect the radioactive seed (Gilcrease et al., 2016), and the pathology staff needs to be aware of how to handle this rare event. Once removed from the breast specimen, the I-125 seed needs to be returned to the nuclear medicine department for storage until returned to the vendor or until it can otherwise be disposed. There are documentation requirements regarding the handling of the 
I-125 seed at each stage of the process. The multidisciplinary aspects of starting an RSL program have been described in detail, starting with identifying a champion through designing and implementing a fail-safe seed tracking system and recovery procedure (Jakub \& Gray, 2015).

A sampling of published reports from academic centers has shown that the positive margin rate using RSL is comparable to that obtained with WL. At the Memorial Sloan-Kettering Cancer Center, the positive margin rate during the initial six months of using the RSL technique was 7.7\% for the 431 RSL patients versus $5.5 \%$ for the 256 WL patients (Murphy, et al., 2013). The University of Texas MD Anderson Cancer Center reported that the combined close or positive margin rate was 20\% (26/127) for the RSL patients and 20\% (104/533) for the WL patients (Dryden, et al., 2016). At the University of Pittsburgh, it was reported that 21.1\% (24/114) of RSL patients required a re-excision for close or positive margins, compared to 26.3\% (31/118) WL patients (Sharek, et al., 2015). The University of Kansas reported a 23\% (10/44) positive margin rate with RSL versus 24\% (15/62) with WL (Rarick, Kimler, \& Tawfick, 2016). Catharina Hospital had one of the lowest positive margin rates, 4.6\% (15/325) with RSL, but there was no corresponding WL group (Van Riet et al., 2010). The Netherlands Cancer Institute (Janssen, et al., 2016) reported positive margin rates with RSL as 9.1\% for DCIS and 9.7\% for invasive cancer, but likewise there was not a comparison WL group. A Canadian multicenter randomized trial also favorably compared RSL to WL, with positive margin rates of $10.5 \%$ for the 152 RSL patients and $11.8 \%$ for the $153 \mathrm{WL}$ patients (Lovrics, et al., 2011). A recent Cochrane Review also concluded that RSL provided comparable outcomes to WL (Chan, Wiseberg-Firtell, Jois, Jensen, \& Audisio, 2015).

Our reported positive margin rates of $12.7 \%$ (14/110) for RSL and 8.5\% (3/25) for WL likewise were not statistically different. Our power calculation revealed if the positive margin rate was $10 \%$ for one technique and $15 \%$ for the other, to detect a difference with $80 \%$ power at the $95 \%$ confidence level required a minimum of 685 patients in each group. To detect a similar difference between a $10 \%$ positive margin with one technique and $20 \%$ for the other required at least 200 patients in each group. Our series, like many of those cited above, lacked a sufficient number of patients to accurately detect a difference of this magnitude.

The difference in close/positive margin rates between the above cited series is partially due to what was considered at each institution to be an adequate surgical margin. The publication of consensus guidelines for stages I and II invasive cancer (Moran et al., 2014) as no tumor on ink and for DCIS (Morrow et al., 2016) as $3 \mathrm{~mm}$ should facilitate comparisons between institutions for future patients. At our institution during the time the patients in our series were treated, we followed the NSABP definition and used no tumor on ink as being a negative margin for both invasive cancer and DCIS.

Patient satisfaction scores have become increasingly important as a quality measure. Two studies have demonstrated higher convenience scores and higher patient satisfaction scores among patients who had breast conserving surgery using RSL rather than wire localization (Hughes et al., 2008), (Bloomquist, Ajkay, Patil, Collett, Frazier, \& Barrio, 2016).

Bleicher et al. (2015) analyzed a Surveillance, Epidemiology, and End Results (SEER)-Medicare cohort consisting of 94,544 women over 66 years of age and determined that the time from diagnosis to surgery correlated with disease specific survival when evaluated in five cohorts $\leq 30,31-60,61-90,91-120$, and 121-180 days, favoring the shorter time from diagnosis to surgery (Bleicher et al., 2015). We examined the time from mammographic abnormality to biopsy and from mammogram to BCS for patients imaged at our facility versus an outside facility. We found there was no difference in the time from mammogram to biopsy for patients imaged at our center versus others $(15.1$ versus 14.6 days, $p$-value $=0.864)$. There was a significant difference in the time from mammogram to BCS for those imaged at our facility versus others (23.0 days versus 35.1 days, $\mathrm{p}$-value $<0.001)$. The SEER-Medicare analysis indicates this difference could have clinical significance.

The analysis of our first 48 RSL patients (Wheeler et al., 2016) indicated the specimen weight was significantly larger for the RSL patients (average of $63 \mathrm{~g}$ ) compared to the WL patients (average of $33 \mathrm{~g}$; $\mathrm{p}=0.02$ ). Since a larger specimen size could cause an inferior cosmetic result, it was encouraging that with the added experience accumulated with the second year of the RSL program, the difference in the specimen size no longer was significant, with a geometric mean of $32.78 \mathrm{~g}$ for the RSL patients compared to 30.26 for the WL patients, p-value 0.553 .

We found no relationship between invasive tumor grade and the likelihood of a positive margin for either grade 1 versus grade 2 or grade 1 versus grade 3. We did find an increased likelihood of having a positive margin for larger tumors. We similarly found no increase likelihood of a positive margin with DCIS nuclear grade, but the larger DCIS lesions were likely to have a positive margin. We did not find an association between the likelihood of a positive margin and the patient's age. 
The Chinese study by Jia reported the increased likelihood of positive margins among patients with Her-2 positive invasive tumors undergoing breast conserving surgery (Jia et al., 2014). We were not able to establish an association between the positive margin rates and the Her-2 copy number or either the ER or PR receptor status.

We started our RSL program February 12, 2014 with one breast surgeon and two diagnostic radiologists trained in this technique. The addition of an additional surgeon and another radiologist did not adversely affect the positive margin rates, but the number of patients was not adequate to detect a difference of even $10 \%$ versus $20 \%$ in the positive margin rates.

\section{Conclusion}

The RSL technique is a convenient alternative to WL of nonpalpable breast lesions. It allows the radioactive localization marker to be conveniently placed up to five days prior to the planned breast conserving surgery. The patient never has a localization wire that could potentially become displaced. The RSL technique capitalizes on the radiologist's skill at placing a marker in the breast at the site of an image guided biopsy, so the placement of the I-125 seed in the breast is relatively straightforward. The RSL technique utilizes the surgeon's skill at using a radiographic probe to remove a sentinel lymph node, adapting it to radioguided removal of the breast specimen. Implementing an RSL program requires multidisciplinary coordination and training of all members of the team and strict adherence to guidelines associated with the use of radioactive materials.

There are numerous publications from academic centers demonstrating that the positive margin rate is not significantly different using RSL and WL, and our series confirms that community hospitals can also adopt the RSL technique with the same expectation. Although our initial experience of our first 48 patients demonstrated a significantly larger specimen size with RSL compared to WL, $63 \mathrm{~g}$ versus $33 \mathrm{~g}$ (p-value $=0.02$ ), this analysis of our first $110 \mathrm{RSL}$ procedures shows no significant difference ( $33 \mathrm{~g}$ RSL versus $30 \mathrm{~g} \mathrm{WL}, \mathrm{p}$-value $=0.553)$. There is no reason to suspect that adoption of the RSL technique will lead to removal of larger breast specimens and an inferior cosmetic result. The few studies available indicate improved patient satisfaction with RSL compared to WL for removal of nonpalpable breast specimens.

Breast surgeons and radiologists in community hospitals should be encouraged to initiate the RSL technique.

Acknowledgement: Special thanks to Victoria Owens, MD, who reviewed the pathology slides when needed to assess the margin distance and other pathologic features.

Table 1. Patient Characteristics

\begin{tabular}{lll}
\hline Stage & WL frequency (\%) & RSL frequency (\%) \\
\hline 0 & $10(28)$ & $24(22)$ \\
I & $18(51)$ & $67(61)$ \\
IIa & $5(14)$ & $12(11)$ \\
IIb & $1(3)$ & $6(5)$ \\
III & $0(0)$ & $1(1)$ \\
IV & $1(3)$ & $0(0)$ \\
\hline Age (years) & WL frequency (\%) & RSL frequency (\%) \\
\hline$<50$ & $2(6)$ & $11(10)$ \\
$50-59$ & $10(28)$ & $36(33)$ \\
$60-69$ & $10(28)$ & $35(32)$ \\
$70-79$ & $8(23)$ & $24(22)$ \\
$80-89$ & $4(11)$ & $4(4)$ \\
$90-99$ & $1(2)$ & $0(0)$ \\
Average age (years) & 65 & 62 \\
\hline \multicolumn{2}{c}{ DCIS component } \\
\hline Median size (mm) & 9.00 & 6.00 \\
Average size (mm) & 3.64 & 11.21 \\
Range (mm) & $1-47$ & $1-50$ \\
\hline \multicolumn{2}{c}{ Invasive Component } \\
\hline Median size (mm) & 13.0 & 11.5 \\
Average size (mm) & 14.44 & 13.35 \\
Range (mm) & $6-33$ & $1-60$ \\
\hline
\end{tabular}

Note. $\mathrm{WL}=$ wire localization; $\mathrm{RSL}=$ radioactive seed localization 
Table 2. Positive margin rate for combined WL and RSL patients by radiologist

\begin{tabular}{lllll}
\hline $\begin{array}{l}\text { Positivie } \\
\text { Margin }\end{array}$ & \multicolumn{5}{c}{ Radiologist } \\
\hline No & Others & A & B & C \\
Yes & 5 & 30 & 48 & 45 \\
Radiologist & 0 & 5 & 3 & 9 \\
& Odds ratio & $95 \%$ CI & p-value \\
& (vs others) & & & \\
\hline A & 1.98 & 0.18 to 274.28 & 0.633 & \\
B & 0.79 & 0.06 to 112.08 & 0.886 & \\
C & 2.30 & 0.24 to 311.03 & 0.545 & \\
\hline
\end{tabular}

Note. $\mathrm{CI}=$ Confidence Interval

\section{References}

Aima, M., Viscariello, N., Patton, T., \& Bednarz, B. (2016). SU-F-J-09: Radioactive seed localization for breast Lumpectomy-towards optimization. Med Phys, 43, 3407. http://dx.doi.org/10.1118/1.4955917

Barentsz, M. W., van den Bosch, M. A., Veldhuis, W., B., van Diest, P. J., Pijnappel, R. M., Witkamp, A. J., \& Verkooijen, H. M. (2013). Radioactive seed localization for non-palpable breast cancer. Br. J. Surg., 100, 582-8. http://dx.doi.org/10.1002/bjs.9068

Bleicher, R. J., Ruth, K., Sigurdson, E. R., Beck, R., Ross, E., Wong, Y-N, ... Egleston, B. L. (2015). Time to surgery and breast cancer survival in the United States. JAMA Oncol, 2, 330-339. http://dx.doi.org/10.1001/jamaoncol.2015.4508

Bloomquist, E. V., Ajkay, N., Patil, S., Collett. A. E., Frazier, T. G., \& Barrio, A., V. (2016). A randomized prospective comparison of patient-assessed satisfaction and clinical outcomes with radioactive seed localization versus wire localization. Breast J, 22, 151-7. http://dx.doi.org/10.1111/tbj.12564

Chan, B. K. Y., Wiseberg-Firtsell, J. A., Jois, R. H. S., Jensen, K., \& Audisio, R. A. (2015). Localization techniques for guided surgical excision of non-palpable breast lesions (Review). The Cochrane Collaboration. http://dx.doi.org/10.1002/14651858/CD009206/pub2

Classic, K. L., Brunette, J. B., \& Carlson, S. K. (2009). Potential for contamination during removal of radioactive seeds from surgically excised tissue. Health Phys, 97, S136-S139.

Darby, S., McGale, P., \& Correa, C. (2011). Effect of radiotherapy after breast-conserving surgery on 10-year recurrence and 15-year breast cancer death: Meta-analysis of individual patient data for 10,801 women in 17 randomized trials. Lancet, 3748, 1707-16. http://dx.doi.org/10.1016/50140-6736(11)62629-2

Davis, P. S., Wechsler, R. J., Feig, S. A., \& March, D. E. (1988). Migration of breast biopsy localization wire. AJR, 150, 787-788.

Dauer, L.T., Thornton, C., Miodownik, D., Boylan, D., Holahan, B., King, V., ... Germain, J. S. (2013). Radioactive seed localization with ${ }^{125}$ I for nonpalpable lesions prior to breast lumpectomy and/or excisional biopsy: methodology, safety, and experience of initial year. Health Physics, 105, 356-365. http://dx.doi.org/10.1097/HP.0b013e31829c03e1

Dryden, M. J., Dogan, B. E., Fox, P., Wang, C., Black, D. M., Hunt, K., \& Yang, W. T. (2016). Imaging factors that Influence surgical margins after preoperative 125|radioactive seed localization of breast lesions: comparison with wire localization. AJR, 206, 1112-118. http://dx.doi.org/10.2214/AJR.15.14715

Gilcrease, M. Z., Dogan, B. E., Black, D. M., Contreras, A., Dryden, M. J., Jimenez, S. M. (2016). Transection of radioactive seeds in breast specimens. Am J Surg Pathol, 40, 1375-9. https://dx.doi.org/10.1097/PAS0000000000000682

Goudreau, S. H., Joseph, J. P., \& Seiler, S. J. (2015). Preoperative radioactive seed localization for nonpalpable breast lesions: techniques, pitfalls, and solutions. RadioGraphics, 35, 1319-1334.

http://dx.doi.org/10.1148/rg.2015140293 
Graham, R. P. D., Jakub, J. W., Brunette, J. J., \& Reynolds, C. (2012). Handling of radioactive seed localization breast specimens in the pathology laboratory. Am J Surg Pathol, 36, 1718-1723. http://dx.doi.org/10.1097/PAS.ob013e318265c37f

Gray, R. J., Salud, C., Nguyen, K., Dauway, E., Friedland, J., Berman, C., ... Cox, C. E. (2001). Randomized prospective evaluation of a novel technique for biopsy or lumpectomy of nonpalpable breast lesions: radioactive seed versus wire localization. Annal Surg Oncol, 8, 711-715.

Homer, M. J. \& Pile-Spellman, E. R. (1986). Needle localization of occult breast lesions with a curved-end retractable wire: technique and pitfalls. Radiology, 161, 547-548. http://dx.doi.org/10.1148/radiology.161.2.3763930

Hughes, J. H., Mason, M. C., Gray, R. J., McLaughlin, S. A., Degnim, A. C., Fulmer, J. T., ... Roarke, M. C. (2008). A multi-site validation trial of radioactive seed localization as an alternative to wire localization. Breast J, 14, 153-7. http://dx.doi.org/10.1111/j.1524-4741.2007.00546.x.

Jakub, J. W., Gray, R. J., Degnim, A. C., Boughey, J. C., Garner, M., \& Cox, C. E. (2010). Current status of radioactive seed for localization on nonpalpable breast lesions. Am J Surg, 199, 522-528. http://dx.doi.org/10.1016/j.amjsurg.2009.05.019

Jakub, J., \& Gray, R. (2015). Starting a radioactive seed localization program. Ann Surg Oncol, 22, 3197-202. http://dx.doi.org/10.1245/s10434-4719-5

Janssen, N. N. Y., Nijkamp, J., Alderliesten,T., Loo, C. E., Rutgers, E. J. T., Sonke, J. J., \& Vranken Peeters, M. T. F. D. (2016). Radioactive seed localization in breast cancer treatment. BJS, 103, 70-80. http://dx.doi.org/10.1002/bjs.9962

Jia, H., Jia, W., Yang, Y., Li, S., Feng, H., Liu, J., ... Su, F. (2014) Her-2 positive breast cancer is associated with an increased risk of positive cavity margins after initial lumpectomy. World Journal of Surgical Oncology, 12, 289-298. http://www.wjso.com/content/12/1/289

Lovrics, P. J., Goldsmith, C. H., Hodgson, N., McCready, D., Gohla, G., Boylan, C., ... Reedijk, M. (2011). A multicentered, randomized, controlled trial comparing radioguided seed localization to standard wire localization for nonpalpable, invasive and in situ breast carcinomas. Ann Surg Oncol, 18, 3407-3414. http://dx.doi.org/10.1245/s10434-011-1699-y

McGhan, L. J., McKeever, S. C., Pockaj, B. A., Wasif, N., Giurescu, M. E., Walton, H. A., \& Gray, R. J. (2011). Radioactive seed localization of nonpalpable breast lesions: review of 1,000 consecutive procedures at a single institution. Ann Surg Oncol, 18, 3096-3101. http://dx.doi.org/10.1245/s10434-011-1910-1

Moran, M. S., Schnitt, S. J., Giuliano, A. E., Harris, J. R., Khan, S. A., Horton, J., ... Morrow, M. (2014). Society of Surgical Oncology-American Society for Radiation Oncology consensus guideline on margins for breast-conserving surgery with whole-breast irradiation in stages I and II invasive breast cancer. Ann Surg Oncol, 21,704-716. http://dx.doi.org.10.1245/s10434-014-3481-4

Morrow, M., Van Zee, K. J., Solin, L.J., Houssami, N., Chavez-MacGregor, M., Harris, J. R., ... Moran, M. S. (2016). Society of Surgical Oncology-American Society for Radiation Oncology-American Society of Clinical Oncology consensus guideline on margins for breast-conserving surgery with whole-breast irradiation in ductal carcinoma in situ. Practical Radiation Oncology, 6, 287-295. http://dx.doi.org/10.1016/j.prro.2016.06.011

Murphy, J. O., Moo, T. A., King, T. A., Van Zee, K. J., Villegas, K. A., Stempel, M., ... Morrow, M. (2013). Radioactive seed localization compared to wire localization in breast-conserving surgery: initial 6-month experience. Ann Surg Oncol, 20, 4121-4127. http://dx.doi.org/10.1245/s10434-013-3166-4

Pavlicek, W., Walton, H. A., Karstaedt, P. J., \& Gray, R. J. (2006). Radiation safety with use of I-125 seeds for localization of nonpalpable breast lesions. Academic Radiology, 13, 909-915. http://dx.doi.org/10.1016/j.acra.2006.03.017

Rarick, J., Kimler, B. R., \& Tawflck, O. (2016). Comparison of margin status and lesional size between radioactive seed localized vs conventional wire localized breast lumpectomy specimens. Ann Diag Path , 21, 47-52. http://dx.doi.org/10.1016/j.anndiagpath.2016.01.003

Sharek, D., Zuley, M. L., Zhang, J. Y., Soran, A., Ahrendt, G. M., \& Ganott, M. A. (2015). Radioactive seed localization versus wire localization for lumpectomies: a comparison of outcomes. AJR, 204, 872-877. http://dx.doi.org/10.2214/AJR.14.12743 
Wheeler, J. A., Harrod, K., Liu, F., Garber, E., Grove-Narayan, L., Moore, A. (2016). Radioactive seed localization for nonpalpable breast lesions in a community hospital setting. CCO 5, 25-30. http://dx.doi.org/10.5539/cco,v5n1p25

VanRiet, Y. E. A., Jansen, F. H., van Beek, M., van de Velde, C. J. H., Rutten, H. J. T., \& Nieuwenhuijzen. (2010). Localization of non-palpable breast cancer using a radiolabelled titanium seed. BJS, 97, 1240-1245. http://dx.doi.org/10.1002/bjs.7097

\section{Copyrights}

Copyright for this article is retained by the author(s), with first publication rights granted to the journal.

This is an open-access article distributed under the terms and conditions of the Creative Commons Attribution license (http://creativecommons.org/licenses/by/4.0/). 